Feature article

\title{
Modification of polypeptide materials by Thiol-X chemistry
}

\author{
Sarah M. Brosnan a, Helmut Schlaad ${ }^{\text {a, b, * }}$ \\ ${ }^{a}$ Max Planck Institute of Colloids and Interfaces, Department of Colloid Chemistry, Research Campus Golm, 14424 Potsdam, Germany \\ ${ }^{\mathrm{b}}$ University of Potsdam, Institute of Chemistry, Karl-Liebknecht-Straße 24-25, 14476 Potsdam, Germany
}

\section{A R T I C L E I N F O}

\section{Article history:}

Received 27 June 2014

Received in revised form 18 August 2014

Accepted 23 August 2014

Available online 6 September 2014

Keywords:

Polypeptide

Thiol-X

Click chemistry

\begin{abstract}
A B S T R A C T
Thiol-X chemistry has proven to be a valuable toolbox for modification of peptides, proteins, monomers, and polymers. Recently, this has become especially true for the modification of polypeptides (monomers or polymers), which has resulted in a plethora of novel polymers and materials. With this in mind, this highlight focuses on the recent literature concerning the modification of polypeptides by the use of thiol$\mathrm{X}$ chemistry, in particular to synthetic polypeptides either at the monomer or polymer stage modified by thiol-ene, -Michael addition, and -yne chemistries.

๑ 2014 Published by Elsevier Ltd. This is an open access article under the CC BY-NC-SA license (http:// creativecommons.org/licenses/by-nc-sa/3.0/).
\end{abstract}

\section{Introduction}

Broadening the utility of manmade materials has been an incredible driving force for the design and utilization of highly effective and efficient chemistries. In particular, in polymer chemistry, the use of "click chemistry" has exploded over the past few decades as a method of choice to take well-studied materials and extend their use beyond their original application [1-3]. The development of such methodologies has transformed how polymer scientists view materials in general: the properties of a monomer, polymer, or material are not set in stone. The ability to work "topdown" (in this case, by modifying existing materials) in addition to "bottom-up" allows researchers quick and easy access to novel materials and material properties. This ability has been particularly significant with biological-based materials and bio-inspired materials, such as polypeptides.

Reactions between thiols and various types of unsaturations or electrophiles, referred to as thiol-X chemistries (Scheme 1) [4], have been used extensively to modify everything from small molecules, polymers, and particles to macroscopic substrates [5,6]. The success of these types of reactions is due to their "click chemistry" nature, as they are highly efficient and selective, produce no or few by-products, and can be conducted under mild reaction conditions. This class of reactions is a highly popular approach to the

\footnotetext{
* Corresponding author. University of Potsdam, Institute of Chemistry, KarlLiebknecht-Straße 24-25, 14476 Potsdam, Germany.

E-mail address: schlaad@uni-potsdam.de (H. Schlaad).
}

modification of biological and biomimetic moieties, as they provide a more biologically friendly approach to the modification of materials. These types of reactions do not require toxic catalysts such as those required in the copper-catalyzed Huisgen [3+2] cycloaddition reaction between azides and alkynes, and they are tolerant to air and water (to a certain extent), which makes them very appealing to provide the facile modification of peptides and proteins. The use of thiol- $X$ chemistry to modify peptides and proteins is not entirely new. Cysteine, a natural amino acid, bears a convenient primary thiol group, making it an ideal substrate for modification in peptides and proteins. This type of chemistry has allowed researchers to expand the application scope of these well studied materials.

More recently, however, much attention has been given to bioinspired materials such as polypeptides [7-10]. Polypeptides are of particular interest because, while they are not natural and thus do not contain signaling information like natural peptides, they do display secondary structures (i.e., $\alpha$-helixes and $\beta$-sheets), self assembly properties, biocompatibility, and biodegradability. Polypeptides are polymerized from amino acid $\mathrm{N}$-carboxyanhydrides (NCAs) [11]; unlike materials synthesized by solid-phase synthesis, polypeptides allow for far greater synthetic diversity and scalability by varying the substituent on the $\alpha$-carbon to the carbonyl, either on the monomer or polymer after polymerization. Polypeptides are not new to polymer chemistry: they were first synthesized in 1906 [12], and much research over the years has been devoted to synthesizing these materials and understanding their structure. 


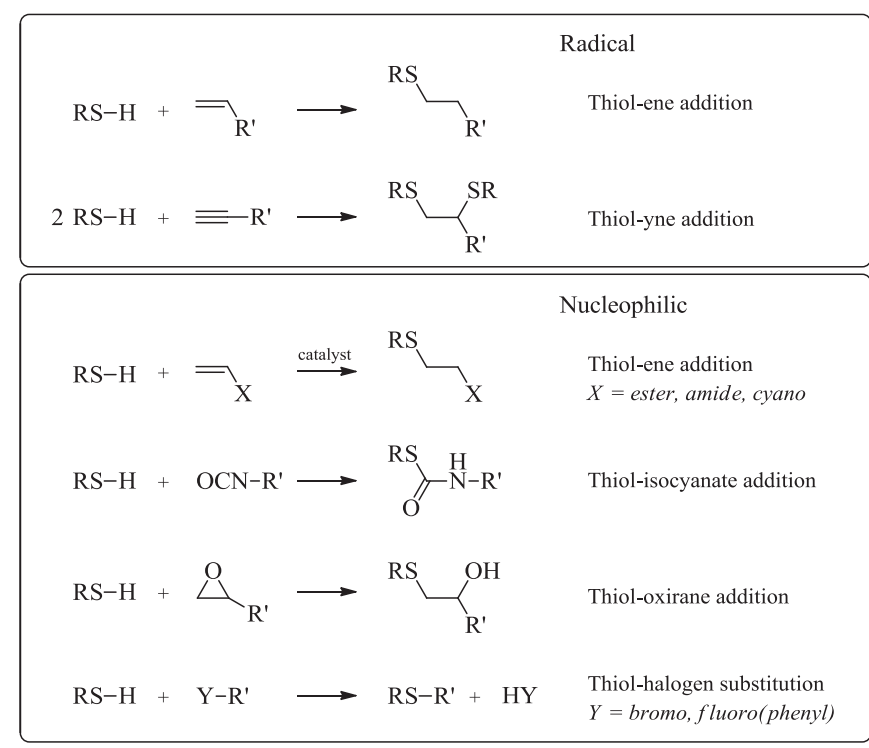

Scheme 1. Radical and nucleophilic thiol-X chemistries.

Recently, the use of thiol-X chemistries on these structures has provided access to a cornucopia of novel monomers, polymers, structures, and materials [13-17]. Many recent reviews discuss the use of polypeptides or thiol-X chemistries, but few focus on the modification of polypeptides by thiol-X chemistries, which is a rapidly growing field. With this in mind, the focus of this feature is a review of the recent use of radical thiol-ene and -yne and nucleophilic thio-Michael addition reactions to modify polypeptide materials.

\section{Thiol-ene}

\subsection{Small peptides and monomer modification}

As a polymer chemist, starting from the bottom up (synthesizing new monomers) is the most natural way to make new materials. If we are thinking about peptides or polypeptides and thiol-ene chemistry, the most natural monomer to start with is cysteine. Cysteine has been used to anchor peptides and proteins to sugars [18], monomers [19], polymers [20-23], hydrogels [24,25] and nanoparticles [26]. The most impressive recent example is the 3D cell microenvironment developed by the Anseth group [24,25] Hydrogels were first formed by crosslinking a tetra-azidefunctionalized polyethylene glycol (PEG) with an enzymatically degradable polypeptide sequence that bared both an activated alkyne (di-fluorinated cyclooctyne, proving both the ring strain and electron withdrawing moieties) for copper-less click chemistry and alkene (Fmoc-Lys(alloc)-OH, protected allyl functionalized lysine) for later thiol-ene chemistry. Once hydrogels with encapsulated cells were obtained under physiological conditions $\left(37^{\circ} \mathrm{C}\right.$ for $\sim 1 \mathrm{~h}$ ), the authors were able to selectively photopattern the gels using traditional photolithographic methods, cytocompatible conditions (365 $\mathrm{nm}$ at $10 \mathrm{~mW} \mathrm{~cm}^{-2}$ ), and a di-fluorescein collagenase-sensitive peptide sequence (an enzymatically degradable peptide that contains two fluoresceins and is terminated by a cysteine unit). They were able to demonstrate this technique with several fluorescently labeled peptides, including a labeled RGD sequence (typically used to enhance cell adhesion to substrates) with good spatial control (on the order of tens of microns) (Fig. 1). This method is a particularly good example of how polypeptides (synthesized by solid-state synthesis) can be attached to substrates

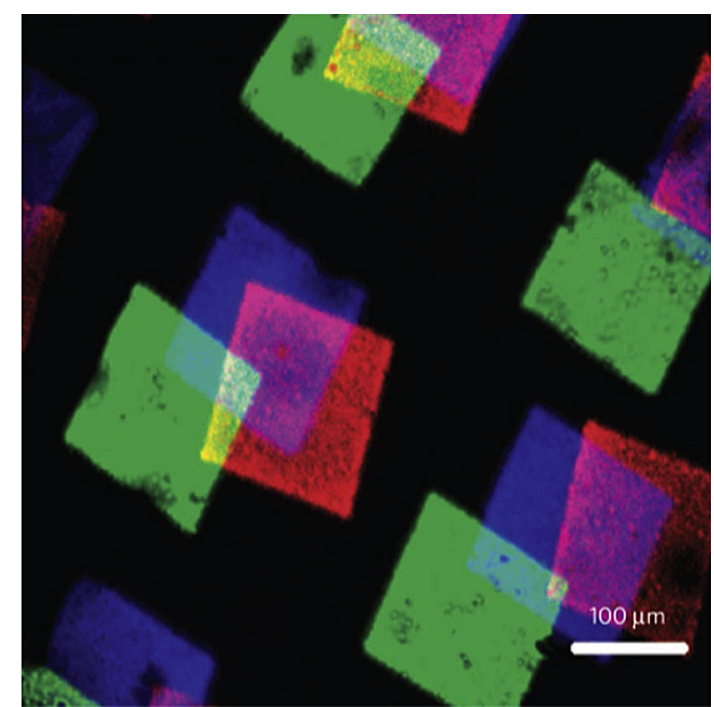

Fig. 1. Hydrogel modified by various fluorescent peptides. Reprinted with permission from Ref. [24] Copyright 2009 Nature Publishing Group.

using mild thiol-ene chemistry conditions, though the extent of functionalization was only observed visually.

Cysteine has also served as the functional precursor for thiolbearing NCAs [21-23]. Das et al. were able to make phosphonated polypeptides from cysteine-derived NCAs that were phosphated by thiol-ene chemistry [21]. Deming and co-workers synthesized and then polymerized a glycosylated L-cysteine NCA and thus achieved water-soluble polymers that formed stable helices [22]. The monomer was synthesized by first benzyloxycarbonyl (Cbz) protecting the L-cysteine and, subsequently, the scission of the disulfide bond to yield the protected cysteine. After separately preparing protected allyl-functionalized galactose or glucose, these sugars were then attached in high yield via thiol-ene chemistry by irradiating a DMF solution containing the Cbzprotected cysteine (2.5 equivalent) and 2,2-dimethoxy-2phenylacetophenone (DMPA, $0.15 \mathrm{~mol} \%$ ) at $365 \mathrm{~nm}$ for $1 \mathrm{~h}$. The NCA was formed by ring closing using $\alpha, \alpha$-dichloromethyl methyl ether and then purified by column chromatography. Polymers of up to $\sim 200$ repeat units with low dispersities $(<1.20)$ were achieved by utilizing $\left(\mathrm{PMe}_{3}\right)_{4} \mathrm{Co}$ as a catalyst, and water soluble polymers modified with galactose and glucose were obtained after deprotection. The authors showed that these materials formed stable helices in water (in DI water and PBS), and when the thioether group on the polymer (or monomer) was oxidized to yield a sulfone the polymer was no longer capable of helical conformations [23].

The above method, however, is slightly tedious in that both the monomer and sugar must be synthesized separately and then polymerized. Additionally, the use of the cobalt catalyst may not be ideal for biological applications. Recently, our group has developed a nearly one-pot method for glycosylation, polymerization, and functionalization (Scheme 2) [27]. Here, instead of a cysteinederived NCA monomer (which avoids any potential ring opening by the thiol) allyl-glycine-derived NCAs were synthesized. The allyl glycine was first ring closed by triphosgene to yield the NCA monomer, and after purification of the NCA, it was then glycosylated using 1-thio- $\beta$-D-glucopyranose-2,3,4,6-tetraacetate while irradiated in the presence of benzophenone in a THF solution. This thiol-ene reaction was conducted in less than stoichiometric amounts, which prevented nucleophilic ring opening of the thiol and had the added advantage of providing excess vinyl groups for further functionalization. In the same flask, polymerization was initiated by hexylamine and reacted for 7 days. Polymers with up to 


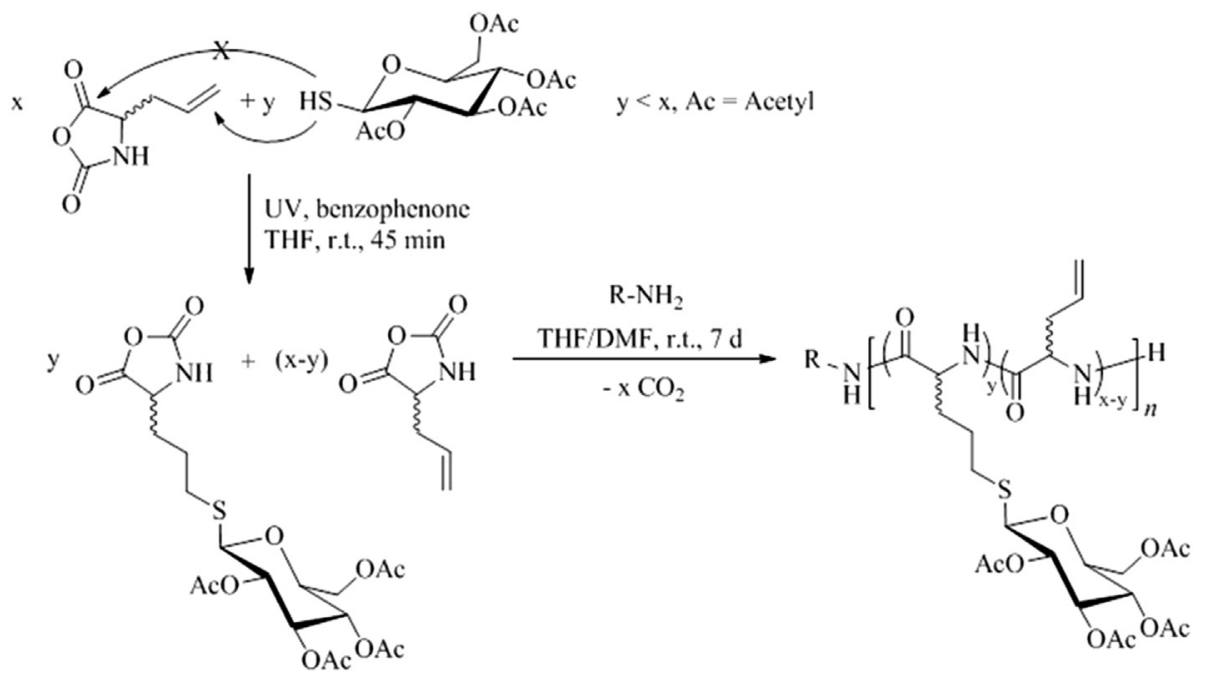

Scheme 2. One-pot functionalization and polymerization of glycosylated polypeptides [27].

$\sim 70 \%$ glycosylated monomer incorporation, 55 repeat units, and reasonable dispersities of $\sim 1.2$ were readily achieved. Lower than expected molecular weight could possibly be attributed to the choice of solvent and the large amount of benzophenone required for functionalization, which was likely required because the light source for the thiol-ene reaction was simply a commercial energysaving bulb. After polymerization, it was demonstrated that the remaining allyl functionalities could be functionalized with a variety of different thiols (e.g., 3-mercaptopropionic acid, $\mathrm{N}$-acetylcysteamine, and thioacetic acid). While further optimization of the method is required, this work demonstrated a more facile and economical method to achieve functional polypeptides.

\subsection{Polymer modification}

\subsubsection{Radical thiol-ene addition}

Likely due to the inherent difficultly of polymerizing bulky and reactive functional groups, most thiol-X chemistry occurs after polymerization [28-33]. Notable recent examples include the work done by the Cheng group [28,29], who have been able to synthesize several polypeptides that have high ionic character, resulting in materials that readily yielded $\beta$-sheets or polymers with high helicity. This group also synthesized serine-derived NCAs (o-penteneL-serine NCAs) that have long carbon spacers between the NCA and allyl functionalities. They were able to polymerize this monomer with PEG and then functionalize (in the polymerization solution, due to solubility limitations of the functionalized polymer) by thiolene chemistry. The functionalization was accomplished by UV irradiation of the polymerization solution with 2-aminoethanethiol hydrochloride (1.7 equivalents) and DMPA (catalytic amounts) for $30 \mathrm{~min}$, though the yield after functionalization was quite low ( $36 \%)$. The resulting polymers formed $\beta$-sheets and had cellpenetrating properties. This group also synthesized a monomer that contained a very long spacer between the terminal vinyl group and the ring. The motivation was that if the chain length between the backbone (which determines the helicity) and the vinyl group (which dictates the solubility) is increased, then it should be possible to create water soluble polypeptides that have high helicity. The researchers were able to accomplish this by polymerization of $\gamma$-(4-allyloxylbenzyl)-L-glutamate NCA with hexamethyldisilazane (HMDS), resulting in well controlled polymerization with up to 100 repeat units by the addition of TBD (1,5,7-triazabicyclo[4.4.0]dec-5-ene) as a co-catalyst, as well as quite low dispersities $(<1.1)$. These polymers were then readily functionalized (quantitatively) within $10 \mathrm{~min}$ by irradiation with UV (365 nm) in DMF/DI water solutions of 2-aminoethanethiol hydrochloride or 3-mercaptopropionic acid (5 equivalents) and Irgacure $^{\circledR} 2959$ (6 wt\%). They were able to achieve helicities up to $\sim 100 \%$ for the 2 -aminoethanethiol hydrochloride (even in $5 \mathrm{M}$ urea) and $82 \%$ for the 3-mercaptopropionic acid modified materials.

Other groups have also sought to make helical water-stable polypeptides using thiol-ene chemistry [30-33]. Tang et al. synthesized diblock copolymers by first polymerizing $\gamma$-allyl-L-glutamic acid-based NCA and then polymerized $\gamma$-3-chloropropanyl-Lglutamate [30]. The resulting block copolymer was first functionalized by transforming the chlorine to an azide; then, copper click chemistry was performed with an alkyne functionalized mannose. The pendant allyl was quantitatively functionalized with 3mercaptopropionic acid (10 equivalents) in water for $60 \mathrm{~min}$ with no photoinitiator added. This yielded a double hydrophilic system, and these polymers displayed both $\alpha$-helical and $\beta$-sheet conformations, depending on the length of the polymer chain.

Our group has recently explored the well-controlled polymerization and co-polymerization of allylglycine and its subsequent functionalization with thiol-ene chemistry [31,32]. Homo- and block copolymers were synthesized by polymerizing allylglycine NCA with hexylamine and PEG-amine (3600 $\mathrm{g} \mathrm{mol}^{-1}$ ) [31]. Functionalization with methyl 3-mercaptopropionate by thiol-ene chemistry was achieved quantitatively by thermal methods (AIBN, $70^{\circ} \mathrm{C}$ for 1 day in DMF). Photochemical addition proved to be less effective, requiring higher concentrations (compared to thermal) to achieve 92\% functionalization with methyl 3mercaptopropionate. The functionalization of sugars proved to be challenging photo-chemically (even with the addition of Irgacure ${ }^{\circledR}$ 819 at 1 equivalent); however, quantitative functionalization of the PEG-poly(allylglycine) with sodium salt of 1 -thio- $\beta$-D-glucopyranose was achieved when the thiol-ene reaction was conducted in trifluoroacetic acid for 2 days (photochemically). This polymer (glycosylated) also proved to form stable water-soluble helices at low $\mathrm{pH}$ [32]. We also explored the glycosylation of $\operatorname{poly}(\gamma$-benzyl-Lglutamate-co-allylglycine) and poly(L-glutamate-co-allylglycine) $[32,33]$. The stimuli responsiveness of these types of block copolymers was tested by synthesizing a series of copolymers that varied in stereochemistry (using poly(L-, D-, or DL-glutamate) and block copolymer ratios). Glycosylation was conducted using 1-thio$\beta$-D-glucopyranose or 1 -thio- $\beta$-D-galactopyranose (1.5 equivalents, 
after treatment to remove the sodium salt) and Irgacure ${ }^{\circledR} 2959$ (0.1 equivalents) or no photoinitiator, in $0.1 \mathrm{M}$ aqueous acetate buffer for $<12 \mathrm{~h}$ to afford quantitative functionalization. These materials show high and reversible helicity at low $\mathrm{pH}$ and high $\mathrm{pH}$, respectfully.

\subsubsection{Thiol-Michael addition}

A less common approach to the modification of polypeptides is the use of thiol-Michael addition reactions. This type of reaction is very common for small molecule or other types of polymer systems, but less typical for the main-chain functionalization of polypeptides [34]. In general, these types of reactions are much more common for bioconjugation (attaching synthetic molecules to biological ones), a recent example of which was shown by Jones et al. [35]. In a one-pot process, they first reduced the disulfide bridge of salmon calcitonin (a 32 amino acid calcitropic hormone) to yield the free thiol (cysteines in nature rarely occur in non-dimer configurations), and they were then were able to react the free thiols with an acrylate-endcapped PEG (7 equivalents, molar mass of $\sim 475 \mathrm{~g} \mathrm{~mol}^{-1}$ ) using tris(2-carboxyethyl)phosphine as the water soluble catalyst in $500 \mathrm{mM}$ PBS buffer ( $\mathrm{pH}$ of 6.5 ) at ambient temperatures for $2 \mathrm{~h}$. The reaction was quantitative (confirmed by MALDI-ToF), and the PEGylated calcitonin retained its original activity (lowering plasma calcium when injected intravenously). This is a good example of a platform that can easily be extended to other biological molecules because of the ease of synthesis.

It is not entirely surprising that the thiol-Michael addition is less common for the modification of polypeptides because of the inherent difficulty in the synthesis of an NCA and the subsequent performance of an NCA polymerization with a Michael acceptor (e.g., acrylate, methylacrylate, and maleimide). Thus, most examples involve the modification of cysteine (either at the monomer or polymer stage). Regardless, there are a few recent examples of using thiol-Michael addition to modify polypeptide monomers and polymers. [36-38]
An interesting example is the work done by $\mathrm{Fu}$ et al., who modified cysteine with various short chain PEGs [36]. The thiolMichael addition was carried out by the addition of either mono acrylate or methylacrylate oligo(ethylene glycol) (1.1 equivalent) to a slightly basic ( $\mathrm{pH}$ of 7.5, synthesized using $1 \mathrm{~N} \mathrm{NaOH}$ ) solution of L-cysteine. Reactions were quantitative, and high yields were obtained for all substrates as long as the solution was kept slightly basic and below a $\mathrm{pH}$ of 8 . The NCA was synthesized by closing the ring with triphosgene, and the oligo(ethylene glycol) modified cysteine NCA was polymerized in THF with triethylamine as the initiator. Due to the long chain and solubility of the NCA, it proved difficult to purify, and the subsequent polymerization was fairly inefficient (even with the use of $\mathrm{Ni}(0)$ complex, primary amine, and HMDS), reaching between 10 and 70\% conversion depending on the length of the PEG (longer chains having the lowest conversions). Regardless, the authors were able to show a reversible temperature switch between $\alpha$-helices and $\beta$-sheet conformations (though this was only significant for the shorter PEG-modified polymers). This same group was also able to demonstrate that when these polymers formed $\beta$-sheets, they formed micelles in water and became random coils when oxidized. [37]

Polymers capable of being Michael acceptors have also been synthesized from vinyl sulfone-substituted L-cysteine NCA to yield polymers with pendant vinyl sulphonates (which are strong Michael acceptors) [38]. This result was accomplished by first modifying L-cysteine (via a thiol-Michael addition) with divinyl sulfone (5 equivalents) at $50{ }^{\circ} \mathrm{C}$ in a methanol solution for $100 \mathrm{~h}$. Uniquely, this reaction resulted in only one addition of the divinyl sulfone, as proven by NMR and electrospray ionization mass spectrometry. The ring was then closed by using $\alpha$-pinene and triphosgene to yield vinyl-sulfone-substituted NCA. The homo- and block copolymers with $\gamma$-benzyl-t-glutamate NCA and $\varepsilon$-carbobenzyloxy-L-lysine NCA were synthesized using 1,1,1-trimethyl- $N$ 2-propenylsilanamine (as the initiator) at $40{ }^{\circ} \mathrm{C}$ in DMF for 2 days (Fig. 2). While they were able to achieve molar masses above

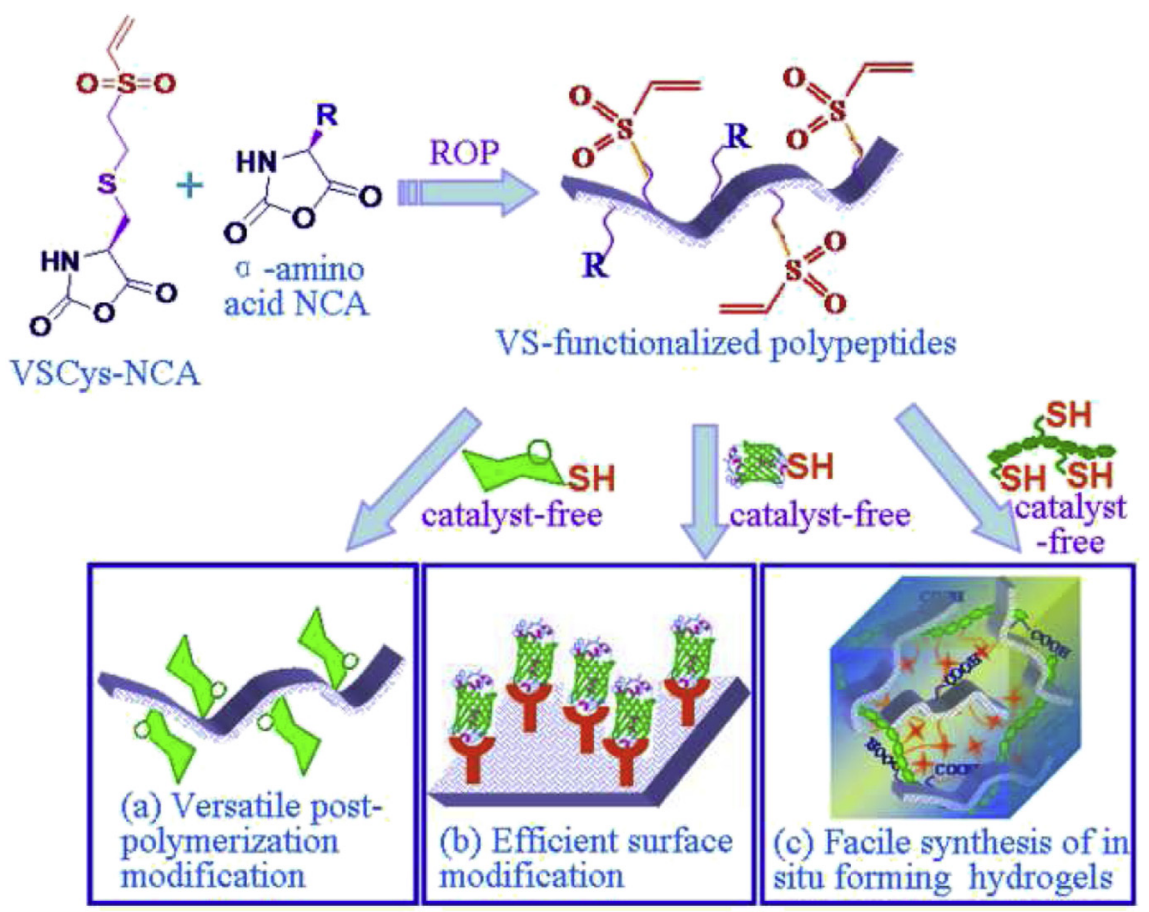

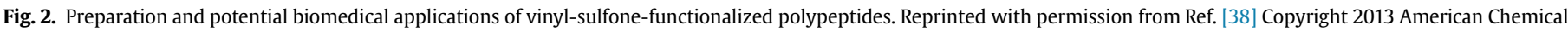
Society. 
$10 \mathrm{~kg} \mathrm{~mol}^{-1}$, the PDIs were rather high for a ring opening reaction (1.20-1.50). Regardless, they were able to functionalize the polymers and materials further with thiols (i.e., 2-mercaptoethanol, 2mercaptoethylamine hydrochloride, L-cysteine, and galactose-SH) in catalyst-free thiol-Michael additions in DMF under a nitrogen atmosphere for 1 day. While the polymers synthesized here were not so precise, this is a particularly unique example, as there are very few polypeptides that are Michael acceptors and that permit catalyst free thiol-Michael addition and a relatively facile functional monomer synthesis.

\section{Thiol-yne}

Complimentary to thiol-ene chemistry, thiol-yne chemistry also has its place in the literature [39-45]. Thiol-yne chemistry works in the same basic way as thiol-ene chemistry but with the added benefit of doubling the functionalization, with the possibility of even heterofunctionalization [39]. This type of chemistry is typically used to end modify, functionalize, and synthesize block copolymers of polypeptides. End modification mainly serves to alter the aggregation properties of the polypeptide [40,41]. Naik et al. used propargyl amine that was first modified by a thiol-yne reaction with dodecanethiol ( 2 equivalents) by UV irradiation (with Irgacure ${ }^{\circledR} 2959$ ) and was then used to initiate the polymerization of $\operatorname{poly}(\gamma$-benzyl-L-glutamate) or poly(Z-lysine) ( $\mathrm{n} \sim 10)$. Alternatively, propargyl amine was used to initiate the polymerization, and the end of the polymer was functionalized under the same conditions. This process resulted in $\mathrm{A}_{2} \mathrm{~B}$ star polymers that readily aggregated in solution [40]. Dong and co-workers synthesized a hyperbranched poly $(\varepsilon-\mathrm{Cbz}-\mathrm{-}$-lysine $)$ by first initiating the polymerization of L-lysine NCA with cystamine and then using 1,4-dithiothreitol to cleave the disulfide bond [41]. The polymer was then end-capped (at the amine end) with 4-pentynoic acid (by DCC/HOBT coupling), resulting in a difunctional end-capped polypeptide with $n \sim 20$ and a moderate PDI of 1.29. Hyperbranched materials were then synthesized by UV irradiating the polymer in DMF in the presence of DMPA (2,2-dimethoxy-phenylacetophenone, $5 \mathrm{wt} \%$ ) for various times. After $30 \mathrm{~min}, \sim 100 \%$ clicking efficiency was achieved without gelation, yielding a molar mass of $21 \mathrm{~kg} \mathrm{~mol}^{-1}$. They also post-modified these hyperbranched materials with a monothiolated PEG using the similar thiol-yne conditions (1.2 equivalents thiol-PEG with $2 \mathrm{wt} \%$ DMPA). Thiol-yne chemistry in general has greater impact on the modification of polymer properties because of the double addition of a functional thiol.
The modification of polypeptides on the main chain via thiolyne also has significant functional advantages over the standard thiol-ene approach [42-45]. The Anseth group showed that cyclic multivalent peptides could be obtained by the reaction of an alkyne-modified short peptide (Lys amino acid) with linear or cyclic RGD peptides, which contain a free cysteine to allow for the thiolyne reaction [42]. These reactions were conducted in either water or DMF with lithium phenyl-2,4,6-trimethylbenzoylphosphinate (LAP) or DMPA, respectfully, and exposed to UV irradiation for 20 min to achieve good yields. Li et al. synthesized an alkynebearing NCA by coupling pentynoic acid with L-glutamic acid in sulfuric acid, followed by ring closure with triphosgene to yield the NCA [43]. This $\gamma$-propargyl-L-glutamate NCA was subsequently polymerized using a mono-aminated PEG as the initiator in DMF for $72 \mathrm{~h}$ at room temperature. This process yielded polymers that were $\sim 20 \mathrm{~kg} \mathrm{~mol}^{-1}$ with dispersities $\sim 1.30$. These polymers were then functionalized with mercaptosuccinic acid (10 equivalents to alkyne) by UV irradiation for 90 min using DMPA (4.5 wt\% of alkyne) as the photoinitiator. This resulted in $69 \%$ thiol functionalization-it is not surprising that the functionalization was lower than what is typical for a thiol-ene reaction because the effect of steric hindrance is significant. Regardless, the authors were able to demonstrate that the functionalization was adequate to complex drugs (i.e., doxorubicin $\mathrm{HCl}$ ) and form vesicles, and the resulting vesicles could potentially be used as drug carriers. Similar work was also done by Huang et al., who additionally reacted the $\operatorname{poly}(\gamma$ propargyl-L-glutamate) with thioglycolic acid, mercaptopropionic acid, and 1-thiogycerol via UV irradiation with DMPA (5 wt\%) and were able to achieve $>90 \%$ conversion within $10 \mathrm{~min}$ [44]. The polymers synthesized here exhibited helical conformations, which may play a large role (the twist of the helix in particular) in achieving high functionalizations. They also observed no single functionalization product, which is not surprising given that the second addition of the thiol should react much faster than the first addition.

Our group has recently developed several approaches to functionalizing alkyne-bearing polypeptides by thiol-yne chemistry [45]. Alkyne-functionalized NCAs were first synthesized by reacting DL-propargylglycine (commercially available) with $\alpha$-pinene and triphosgene to yield the alkyne monomer (though in low yield). Copolymers were synthesized using either hexylamine or benzylamine with $\gamma$-benzyl-L-glutamate NCA as the initiator to produce statistical copolymers. Polymers with $>10 \mathrm{~kg} \mathrm{~mol}^{-1}$ with low dispersities $(<1.20)$ were readily achieved (Fig. 3$)$. The poly $(\gamma$-benzyl-
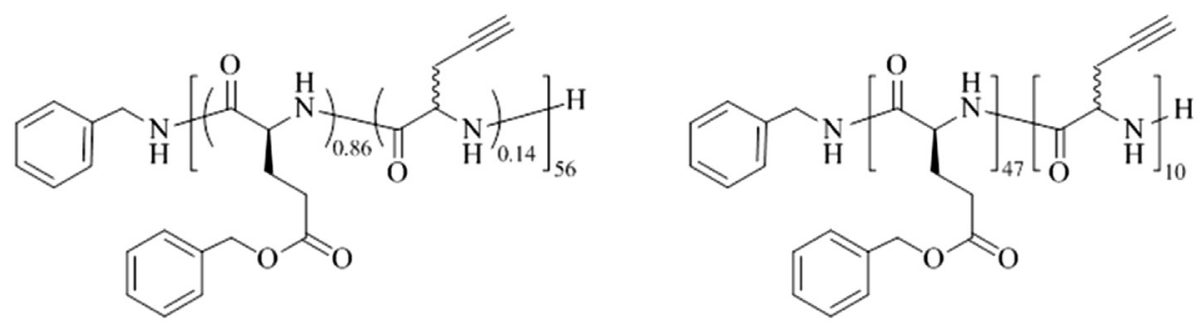<smiles>CC(=O)OC[C@H]1O[C@H](S)[C@@H](OC(C)=O)[C@H](OC(C)=O)[C@@H]1O</smiles><smiles>Cc1cc(=O)oc2cc(S)ccc12</smiles><smiles>COC(=O)CCS</smiles>

Fig 3. Thiol-yne functionalizable polypropargylglycine (statistical and block) copolymers. 
L-glutamate-co-propargylglycine) polymers were functionalized with 1-thio- $\beta$-D-glucose-2,3,4,6-tetraacetate, $\quad 7$-mercapto-4methylcoumarin, and methyl-3-mercaptopropionate via irradiation by either a medium-pressure mercury UV lamp or a 26 W UVB lamp for $40 \mathrm{~h}$. Similar to other reports, however, it was not possible to fully functionalize the alkyne, likely due to steric hindrance, but unlike previous reports, the single addition was possible (except for the addition of the coumarin derivative, which retains unreacted alkynes). This is rather unique, considering that the vinyl sulfide is more reactive than the alkyne to thiol-ene chemistry. These polymers were also deprotected and then functionalized with 1 -thio- $\beta$ D-glucopyranose in $0.1 \mathrm{M}$ acetate buffer and then irradiated overnight. The resulting glycosylated polymers demonstrated greater helicity than the non-functionalized polypeptide (an increase from $24 \%$ to $41 \%$ ), possible due to increased water solubility and side chain hydrogen bonding interactions.

\section{Conclusion/perspective}

The ability to functionalize biological and bio-inspired materials is critical to expanding the overall utility of well known materials, such as peptides and polypeptides. The use of thiol-X chemistry should continue to be critical to this goal because of its biology friendly, facile, and simple methodology. Continuing work should include raising the yield and extent of functionalization for more complicated substrates (long chain sugars and peptides) and thiolyne reactions. Simplification of monomer synthesis and purification of NCAs are critical areas of need, as these are the highest barriers to the commercialization and general use of these materials. Future applications should likely expand to unique materials, such as stimuli-responsive films, that are biocompatible, biodegradable, and easy to synthesize. The future is indeed very bright for this topic, which will likely continue to become more significant over the next decade.

\section{Acknowledgments}

The authors gratefully acknowledge the following funding sources, National Science Foundation Postdoctoral Research Fellowship in Biology under Grant No. DBI-1308104 and Max Planck Society.

\section{References}

[1] Iha RK, Wooley KL, Nyström AM, Burke DJ, Kade MJ, Hawker CJ. Chem Rev 2009;109:5620.

[2] Sumerlin BS, Vogt AP. Macromolecules 2010;43:1.

[3] Karmann L, Kazmaier U. Eur J Org Chem 2013:7101.

[4] Ten Brummelhuis N, Schlaad H. Radical thiol-X chemistry. In: Chatgilialoglu C, Studer A, editors. Encyclopedia of radicals in chemistry, biology and materials, vol. 4. Chichester: John Wiley \& Sons Ltd; 2012. p. 2017-56.

[5] Lowe AB. Polym Chem 2010;1:17.

[6] Lowe AB. Polym Chem 2014:5:4820.

[7] Ouadir MA, Martin M, Hammond PT. Chem Mater 2014:26:461.

[8] Canalle LA, Lowik DWPM, van Hest JCM. Chem Soc Rev 2010;39:329.

[9] Huang J, Heise A. Chem Soc Rev 2013;42:7373.

[10] Kramer JR, Deming TJ. Polym Chem 2014:5:671.

[11] Kricheldorf HR. Angew Chem Int Ed 2006;45:5752.
[12] Leuchs H. Ber Dtsch Chem Ges 1906;39:857.

[13] Kade MJ, Burke DJ, Hawker CJ. J Polym Sci A Polym Chem 2010;48:743.

[14] Hoyle CE, Bowman CN. Angew Chem Int Ed 2010;49:1540.

[15] Massia A, Nanni D. Org Biomol Chem 2012;10:3791.

[16] Hoyle CE, Lee TY, Roper T. J Polym Sci A Polym Chem 2004;42:5301.

[17] Chan JW, Hoyle CE, Lowe AB, Bowman M. Macromolecules 2010;43:6381.

[18] Dondoni A, Massi A, Nanni P, Roda A. Chem Eur J 2009;15:11444.

[19] Xiao J, Tolbert TJ. Biomacromolecules 2009;10:1939.

[20] Habraken GJM, Koning CE, Heuts JPA, Heise A. Chem Commun 2009:3612.

[21] Das S, Kar M, Gupta SS. Polym Chem 2013;4:4087.

[22] Kramer JR, Deming TJ. J Am Chem Soc 2012;134:4112.

[23] Kramer JR, Deming TJJ. Am Chem Soc 2014;136:5547.

[24] DeForest CA, Polizzotti BD, Anseth KS. Nat Mater 2009;8:659.

[25] Fairbanks BD, Schwartz MP, Halevi AE, Nuttelman CR, Bowman CN, Anseth KS. Adv Mater 2009;21:5005.

[26] Drappier C, Wirotius AL, Bathany K, Ibarboure E, Condassamy O, Garanger E et al. Polym Chem 2013;4:2011.

[27] Krannig KS, Doriti A, Schlaad H. Macromolecules 2014;47:2536.

[28] Zhang Y, Lu H, Lin Y, Cheng J. Macromolecules 2011;44:6641.

[29] Tang H, Yin L, Lu H, Cheng J. Biomacromolecules 2012;13:2609.

[30] Tang H, Zhang D. Polym Chem 2011;2:1542.

[31] Sun J, Schlaad H. Macromolecules 2010;43:4445.

32] Krannig KS, Sun J, Schlaad H. Biomacromolecules 2014:15:978.

[33] Krannig KS, Schlaad H. J Am Chem Soc 2012;134:18542.

[34] Nair DP, Podgórski M, Chatani S, Gong T, Xi W, Fenoli CR, et al. Chem Mater 2014;26:724.

[35] Jones MW, Mantovani G, Ryan SM, Wang X, Brayden DJ, Haddleton DM. Chem Commun 2009:5272.

[36] Fu X, Shen Y, Fu W, Li Z. Macromolecules 2013;46:3753.

[37] Fu X, Ma Y, Shen Y, Fu W, Li Z. Biomacromolecules 2014:15:1055.

[38] Conte Lo, Pacifico M, Chambery S, Marra A, Dondoni A. A. J Org Chem 2010;75: 4644.

[39] Zhou J, Chen P, Deng C, Meng F, Cheng R, Zhong Z. Macromolecules 2013;46: 6723.

[40] Naik SS, Chan JW, Comer C, Hoyle CE, Savin DA. Polym Chem 2011;2:303.

[41] Chang X, Dong CM. Biomacromolecules 2013;14:3329.

[42] Aimetti AA, Feaver KR, Anseth KS. Chem Commun 2010;46:5781.

[43] Li M, Lv S, Tang Z, Song W, Yu H, Sun H, et al. Macromol Biosci 2013;13:1150.

[44] Huang Y, Zeng Y, Yang J, Zeng Z, Zhu F, Chen X. Chem Commun 2011;47:7509.

[45] Krannig KS, Huang J, Heise A, Schlaad H. Polym Chem 2013;4:3981.

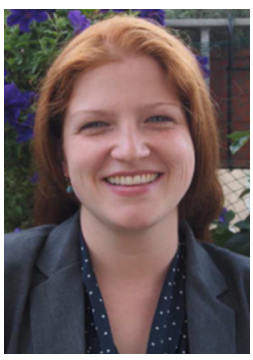

Sarah M. Brosnan received her B.S. in chemistry from the University of Massachusetts Amherst while conducting research in Prof. E. Bryan Coughlin's group. She then went on to receive her Ph.D. at the University of North Carolina at Chapel Hill under the supervision of Prof. Valerie Sheares Ashby in 2012, were she developed novel biomaterials for unique applications. Currently, she is an NSF post-doctoral fellow working at the Max Planck Institute for Colloids and Interfaces under the supervision of Prof. Markus Antonietti and Prof. Helmut Schlaad.

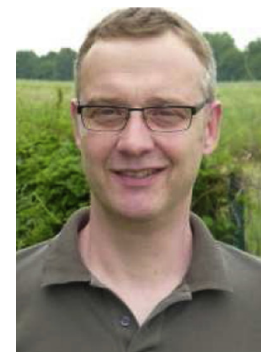

Helmut Schlaad studied chemistry at the University of Mainz, Germany, and earned a doctoral degree in Physical Chemistry, under Axel H. E. Müller in 1997. After a one year post-doctoral fellowship with Rudolf Faust at the University of Massachusetts in Lowell, USA, he moved to the Max Planck Institute of Colloids and Interfaces in Potsdam, Germany. He finished habilitation, mentored by Markus Antonietti, and became senior scientist in 2004. He recently joined the University of Potsdam as Professor (W2) of Polymer Chemistry. His research interests are directed towards polymer synthesis, smart functional materials, and bioinspired structures based on synthetic and biohybrid polymers. 